\title{
A modified two-sided approximation method for a four-point Vallée-Poussin type problem
}

\author{
O. Pytovka
}




\title{
A MODIFIED TWO-SIDED APPROXIMATION METHOD FOR A FOUR-POINT VALLÉE-POUSSIN TYPE PROBLEM
}

\author{
O. PYTOVKA
}

Received 24 September, 2008

\begin{abstract}
We develop a modified two-sided approximation method for a four-point boundary value problem of the Vallée-Poussin type for a system of non-linear differential equations of fourth order with argument deviations.
\end{abstract}

2000 Mathematics Subject Classification: 65L10, 34K10, 34K28

Keywords: two-sided approximation method, Vallée-Poussin problem, differential inequality, comparison function

\section{INTRODUCTION}

There are many works dealing with constructive methods for approximate integration of boundary value problems for ordinary differential equations, which allow one to obtain a direct algorithm to error estimation (see, e. g., $[4,10,11]$ and references therein). These methods include the two-sided methods, which give provide a possibility to construct approximate solutions and, on every step of iteration, obtain a posteriori error estimates of the successive approximations. Numerous research papers are devoted to the construction of new modifications of two-sided methods aimed at the study of various boundary value problems for ordinary differential equations (see, e. g., $[1-3,9]$.

This paper is devoted to the investigation of a four-point boundary-value problem of the Vallée-Poussin type for a system of non-linear differential equations with argument deviation by using a suitable version of the two-sided method generalising the works $[5,6]$.

\section{Problem Settings, DEFInitions AND NOtATIONS}

Let us consider the following problem of Vallée-Poussin's type: to find a solution $Y=\left(y_{i}\right)_{i=1}^{n}$ of the system of differential equations

$$
Y^{(4)}(x)=F\left(x, Y(x),\left(\mathcal{g}_{\Lambda} Y\right)(x),\left(\mathcal{g}_{\Theta} Y\right)(x)\right), \quad x \in[0, \ell],
$$


which satisfies the conditions

$$
Y(0)=A_{1}, \quad Y(\ell / 3)=A_{2}, \quad Y(2 \ell / 3)=A_{3}, \quad Y(\ell)=A_{4},
$$

and

$$
Y(x)= \begin{cases}\Phi(x) & \text { if } x \in\left[\lambda_{0}, 0\right], \\ \Psi(x) & \text { if } x \in\left[\ell, \theta_{0}\right],\end{cases}
$$

where $F:[0, \ell] \times \mathbb{R}^{3 n} \rightarrow \mathbb{R}^{n}$, the vector-functions $\Lambda=\left(\lambda_{i}\right)_{i=1}^{n}$ and $\Theta=\left(\theta_{i}\right)_{i=1}^{n}$ from $C\left([0, \ell], \mathbb{R}^{n}\right)^{*}$ are such that $\lambda_{i}(x) \leq x, \theta_{i}(x) \geq x$ for all $x \in[0, \ell], i=\overline{1, n}$, $\lambda_{0}:=\min \left\{\lambda_{i}(x) \mid x \in[0, \ell], i=\overline{1, n}\right\}, \quad \theta_{0}:=\max \left\{\theta_{i}(x) \mid x \in[0, \ell], i=\overline{1, n}\right\}$, and $A_{s}=\left(a_{i s}\right)_{i=1}^{n} \in \mathbb{R}^{n}$ for $s=\overline{1,4}$, and $\Phi \in C\left(\left[\lambda_{0}, 0\right], \mathbb{R}^{n}\right), \Psi \in C\left(\left[\ell, \theta_{0}\right], \mathbb{R}^{n}\right)$ are given initial vector-functions satisfying the conditions

$$
\Phi(0)=A_{1}, \quad \Psi(\ell)=A_{4} .
$$

The operator $\mathcal{F}_{\Gamma}: C\left(\left[\lambda_{0}, \theta_{0}\right], \mathbb{R}^{n}\right) \rightarrow C\left([0, \ell], \mathbb{R}^{n}\right)$ appearing in (2.1) is defined by the formula

$$
\left(\mathcal{L}_{\Gamma} Y\right)(x):=\left(y_{i}\left(\gamma_{i}(x)\right)\right)_{i=1}^{n}, \quad x \in[0, \ell],
$$

for any $\Gamma=\left(\gamma_{i}\right)_{i=1}^{n} \in C\left([0, \ell], \mathbb{R}^{n}\right)$ and $Y=\left(y_{i}\right)_{i=1}^{n} \in C\left(\left[\lambda_{0}, \theta_{0}\right], \mathbb{R}^{n}\right)$.

\section{Assumptions}

In the sequel, let us suppose that the right-hand side $F:[0, \ell] \times \mathscr{D}^{3} \rightarrow \mathbb{R}^{n}, \mathscr{D} \subseteq \mathbb{R}^{n}$, of the equation (2.1) belongs to the class $\mathcal{M}_{\mathscr{D}}([0, \ell])$, where $\mathcal{M}_{\mathscr{D}}([0, \ell])$ denotes the set of the vector-functions $F$ satisfying the following conditions:

(1) $F \in C\left([0, \ell] \times \mathscr{D}^{3}, \mathbb{R}^{n}\right)$;

(2) there exists a vector-function $H \in C\left([0, \ell] \times \mathscr{D}^{6}, \mathbb{R}^{n}\right)$ such that:

(a) the equality

$$
H(x, U, U)=F(x, U)
$$

holds for all $x \in[0, \ell]$ and $U \in \mathscr{D}^{3}$;

(b) the inequality

$$
\begin{aligned}
& H\left(x, P_{1}(x),\left(\mathcal{g}_{\Lambda} P_{1}\right)(x),\left(\mathcal{g}_{\Theta} P_{1}\right)(x), Q_{2}(x),\left(\mathcal{g}_{\Lambda} Q_{2}\right)(x),\left(\mathcal{g}_{\Theta} Q_{2}\right)(x)\right) \\
& \geq H\left(x, Q_{1}(x),\left(\mathcal{g}_{\Lambda} Q_{1}\right)(x),\left(\mathcal{g}_{\Theta} Q_{1}\right)(x), P_{2}(x),\left(\mathcal{g}_{\Lambda} P_{2}\right)(x),\left(\mathcal{g}_{\Theta} P_{2}\right)(x)\right)
\end{aligned}
$$

is satisfied for all $x \in[0, \ell]$ and every vector-functions $P_{k}, Q_{k}:\left[\tau_{0}, \theta_{0}\right] \rightarrow$ $\mathbb{R}^{n}, k=1,2$, whose restrictions on $[0, \ell]$ belong to $C^{4}\left([0, \ell], \mathbb{R}^{n}\right)$, such that $P_{k}(x), Q_{k}(x) \in \mathscr{D}$ for all $x \in\left[\lambda_{0}, \theta_{0}\right], k=1,2$, and

$$
\begin{aligned}
& P_{k}(x) \leq Q_{k}(x) \quad \text { for } x \in[0, \ell / 3] \cup[2 \ell / 3, \ell], k=1,2 \text {, } \\
& P_{k}(x) \geq Q_{k}(x) \quad \text { for } x \in[\ell / 3,2 \ell / 3], k=1,2 \text {, } \\
& P_{k}^{(4)}(x) \geq Q_{k}^{(4)}(x) \quad \text { for } x \in[0, \ell], k=1,2 .
\end{aligned}
$$

${ }^{*} C\left([0, \ell], \mathbb{R}^{n}\right)$ is the usual Banach space of continuous vector-functions from $[0, \ell]$ to $\mathbb{R}^{n}$. 
(c) the vector-function $H$ satisfies the Lipschitz condition with a non-negative matrix $K=\left(k_{i j}\right)_{i, j=1}^{n}$, i.e.,

$$
\begin{array}{r}
\left|H\left(x, P_{10}, P_{11}, P_{12}, Q_{10}, Q_{11}, Q_{12}\right)-H\left(x, P_{00}, P_{01}, P_{02}, Q_{00}, Q_{01}, Q_{02}\right)\right| \\
\leq K\left(\sum_{s=0}^{2}\left(\left|P_{1 s}-P_{0 s}\right|+\left|Q_{1 s}-Q_{0 s}\right|\right)\right),
\end{array}
$$

for all $P_{s 0}, P_{s 1}, P_{s 2}, Q_{s 0}, Q_{s 1}, Q_{s 2}$ from $\mathcal{D}, s=0,1$, and all $x \in[0, \ell]$.

In (3.1), (3.2), and all similar relations below, the inequalities between vectors and the absolute value sign are understood component-wise.

\section{PReliminary considerations}

Due to the fact that the corresponding linearised homogeneous boundary value problem has only the trivial solution on $[0, \ell]$, the solution $Y$ of problem (2.1)-(2.3) can be represented in the form

$$
Y(x)= \begin{cases}\Phi(x) & \text { for } x \in\left[\lambda_{0}, 0\right], \\ \Omega(x)-\left(\mathcal{T} F\left(\cdot, Y(\cdot),\left(\mathcal{I}_{\Lambda} Y\right)(\cdot),\left(\mathcal{L}_{\Theta} Y\right)(\cdot)\right)\right)(x) & \text { for } x \in[0, \ell], \\ \Psi(x) & \text { for } x \in\left[\ell, \theta_{0}\right],\end{cases}
$$

where the vector-function $\Omega(x)=\left(\omega_{i}(x)\right)_{i=1}^{n}$ has the components

$$
\omega_{i}(x)=a_{i 1}+\frac{243}{4 \ell^{6}}\left|\begin{array}{cccc}
x & 0 & x^{2} & x^{3} \\
\frac{\ell}{3} & a_{i 2}-a_{i 1} & \frac{\ell^{2}}{9} & \frac{\ell^{3}}{27} \\
\frac{2 \ell}{3} & a_{i 3}-a_{i 1} & \frac{4 \ell^{2}}{9} & \frac{8 \ell^{3}}{27} \\
\ell & a_{i 4}-a_{i 1} & \ell^{2} & \ell^{3}
\end{array}\right|, \quad x \in[0, \ell],
$$

the operator $\mathcal{T}: C\left([0, \ell], \mathbb{R}^{n}\right) \rightarrow C\left([0, \ell], \mathbb{R}^{n}\right)$ for any $Z \in C\left([0, \ell], \mathbb{R}^{n}\right)$ is defined by the formula

$$
(\mathcal{T} Z)(x):=\frac{81}{8 \ell^{6}} \int_{0}^{\ell} g(x, \xi) Z(\xi) d \xi, \quad x \in[0, \ell]
$$

and $g$ is the Green function $[7,8]$ of the problem given by the relations

$$
\begin{aligned}
& g(x, \xi)=\left\{\begin{array}{l}
g_{1}(x, \xi), 0 \leq x \leq \frac{\ell}{3}, \\
g_{2}(x, \xi), \frac{\ell}{3} \leq x \leq \frac{2 \ell}{3}, \\
g_{3}(x, \xi), \frac{2 \ell}{3} \leq x \leq \ell,
\end{array} \quad g_{1}(x, \xi)=\left\{\begin{array}{l}
R_{11}(x, \xi), 0 \leq \xi \leq x, \\
R_{12}(x, \xi), x \leq \xi \leq \frac{\ell}{3}, \\
R_{13}(x, \xi), \frac{\ell}{3} \leq \xi \leq \frac{2 \ell}{3}, \\
R_{14}(x, \xi), \frac{2 \ell}{3} \leq \xi \leq \ell,
\end{array}\right.\right. \\
& g_{2}(x, \xi)=\left\{\begin{array}{l}
R_{21}(x, \xi), 0 \leq \xi \leq \frac{\ell}{3}, \\
R_{22}(x, \xi), \frac{\ell}{3} \leq \xi \leq x, \\
R_{23}(x, \xi), x \leq \xi \leq \frac{2 \ell}{3}, \\
R_{24}(x, \xi), \frac{2 \ell}{3} \leq \xi \leq \ell,
\end{array} \quad g_{3}(x, \xi)=\left\{\begin{array}{l}
R_{31}(x, \xi), 0 \leq \xi \leq \frac{\ell}{3}, \\
R_{32}(x, \xi), \frac{\ell}{3} \leq \xi \leq \frac{2 \ell}{3}, \\
R_{33}(x, \xi), \frac{2 \ell}{3} \leq \xi \leq x, \\
R_{34}(x, \xi), x \leq \xi \leq \ell,
\end{array}\right.\right.
\end{aligned}
$$




$$
R_{k 1}(x, \xi)=\left|\begin{array}{cccc}
x & (x-\xi)^{3} & x^{2} & x^{3} \\
\frac{\ell}{3} & \left(\frac{\ell}{3}-\xi\right)^{3} & \frac{\ell^{2}}{9} & \frac{\ell^{3}}{27} \\
\frac{2 \ell}{3} & \left(\frac{2 \ell}{3}-\xi\right)^{3} & \frac{4 \ell^{2}}{9} & \frac{8 \ell^{3}}{27} \\
\ell & (\ell-\xi)^{3} & \ell^{2} & \ell^{3}
\end{array}\right|, R_{k 4}(x, \xi)=\left|\begin{array}{cccc}
x & 0 & x^{2} & x^{3} \\
\frac{\ell}{3} & 0 & \frac{\ell^{2}}{9} & \frac{\ell^{3}}{27} \\
\frac{2 \ell}{3} & 0 & \frac{4 \ell^{2}}{9} & \frac{8 \ell^{3}}{27} \\
\ell & (\ell-\xi)^{3} & \ell^{2} & \ell^{3}
\end{array}\right|
$$

for $k=\overline{1,3}$,

$$
R_{12}(x, \xi)=\left|\begin{array}{cccc}
x & 0 & x^{2} & x^{3} \\
\frac{\ell}{3} & \left(\frac{\ell}{3}-\xi\right)^{3} & \frac{\ell^{2}}{9} & \frac{\ell^{3}}{27} \\
\frac{2 \ell}{3} & \left(\frac{2 \ell}{3}-\xi\right)^{3} & \frac{4 \ell^{2}}{9} & \frac{8 \ell^{3}}{27} \\
\ell & (\ell-\xi)^{3} & \ell^{2} & \ell^{3}
\end{array}\right|, R_{33}(x, \xi)=\left|\begin{array}{cccc}
x & (x-\xi)^{3} & x^{2} & x^{3} \\
\frac{\ell}{3} & 0 & \frac{\ell^{2}}{9} & \frac{\ell^{3}}{27} \\
\frac{2 \ell}{3} & 0 & \frac{4 \ell^{2}}{9} & \frac{8 \ell^{3}}{27} \\
\ell & (\ell-\xi)^{3} & \ell^{2} & \ell^{3}
\end{array}\right|
$$

and

$$
\begin{aligned}
& R_{22}(x, \xi)=R_{32}(x, \xi)=\left|\begin{array}{cccc}
x & (x-\xi)^{3} & x^{2} & x^{3} \\
\frac{\ell}{3} & 0 & \frac{\ell^{2}}{9} & \frac{\ell^{3}}{27} \\
\frac{2 \ell}{3} & \left(\frac{2 \ell}{3}-\xi\right)^{3} & \frac{4 \ell^{2}}{9} & \frac{8 \ell^{3}}{27} \\
\ell & (\ell-\xi)^{3} & \ell^{2} & \ell^{3}
\end{array}\right|, \\
& R_{13}(x, \xi)=R_{23}(x, \xi)=\left|\begin{array}{cccc}
x & 0 & x^{2} & x^{3} \\
\frac{\ell}{3} & 0 & \frac{\ell^{2}}{9} & \frac{\ell^{3}}{27} \\
\frac{2 \ell}{3} & \left(\frac{2 \ell}{3}-\xi\right)^{3} & \frac{4 \ell^{2}}{9} & \frac{8 \ell^{3}}{27} \\
\ell & (\ell-\xi)^{3} & \ell^{2} & \ell^{3}
\end{array}\right| .
\end{aligned}
$$

It is easy to see that

$$
g_{1}(x, \xi) \geq 0, \quad g_{2}(x, \xi) \leq 0, \quad g_{3}(x, \xi) \geq 0 \quad \text { for }(x, \xi) \in[0, \ell] \times[0, \ell] .
$$

Definition. Vector-functions $Z_{0}, V_{0}:\left[\lambda_{0}, \theta_{0}\right] \rightarrow \mathscr{D}$ whose restrictions on $[0, \ell]$ belong to the space $C^{4}\left([0, \ell], \mathbb{R}^{n}\right)$ are called comparison functions of problem (2.1)(2.3) if they satisfy the boundary conditions (2.2), the initial condition (2.3), and the inequalities

$$
\begin{array}{ll}
Z_{0}(x) \leq V_{0}(x) & \text { for } x \in[0, \ell / 3] \cup[2 \ell / 3, \ell] \\
Z_{0}(x) \geq V_{0}(x) & \text { for } x \in[\ell / 3,2 \ell / 3] .
\end{array}
$$

Notation. For any vector-functions $P, Q:\left[\lambda_{0}, \theta_{0}\right] \rightarrow \mathbb{R}^{n}$ we set $\langle P, Q\rangle=\left\{u \in \mathbb{R}^{n} \mid \min \{P(x), Q(x)\} \leq u \leq \max \{P(x), Q(x)\}\right.$ for some $\left.x \in\left[\lambda_{0}, \theta_{0}\right]\right\}$, where the operations "min" and "max" for vectors are understood component-wise. 


\section{CONSTRUCTION OF THE ALTERNATIVE TWO-SIDED METHOD FOR PROBLEM}

$$
\text { (2.1)-(2.3) }
$$

Let us construct the successive approximations $\left\{Z_{p}\right\}_{p=1}^{\infty}$ and $\left\{V_{p}\right\}_{p=1}^{\infty}$ of a solution of problem (2.1)-(2.3) according to the formulae

$$
\begin{gathered}
Z_{p+1}(x)= \begin{cases}\Phi(x) & \text { for } x \in\left[\lambda_{0}, 0\right], \\
\Omega(x)-\left(\mathcal{T} F_{p}\right)(x) & \text { for } x \in[0, \ell], \\
\Psi(x) & \text { for } x \in\left[\ell, \theta_{0}\right],\end{cases} \\
V_{p+1}(x)= \begin{cases}\Phi(x) & \text { for } x \in\left[\lambda_{0}, 0\right], \\
\Omega(x)-\left(\mathcal{T} F^{p}\right)(x) & \text { for } x \in[0, \ell], \\
\Psi(x) & \text { for } x \in\left[\ell, \theta_{0}\right],\end{cases}
\end{gathered}
$$

where

$$
\begin{aligned}
F^{p}(x) & =H\left(x, Z_{p}(x),\left(\mathcal{L}_{\Lambda} Z_{p}\right)(x),\left(\mathcal{L}_{\Theta} Z_{p}\right)(x), V_{p}(x),\left(\mathcal{L}_{\Lambda} V_{p}\right)(x),\left(\mathcal{L}_{\Theta} V_{p}\right)(x)\right), \\
F_{p}(x) & =H\left(x, V_{p}(x),\left(\mathcal{L}_{\Lambda} V_{p}\right)(x),\left(\mathcal{L}_{\Theta} V_{p}\right)(x), Z_{p}(x),\left(\mathcal{L}_{\Lambda} Z_{p}\right)(x),\left(\mathcal{L}_{\Theta} Z_{p}\right)(x)\right)
\end{aligned}
$$

for all $x \in[0, \ell]$, and the zero approximations $Z_{0}$ and $V_{0}$ are comparison functions of problem (2.1)-(2.3) satisfying the conditions

$$
\begin{aligned}
& \alpha_{0}(x):=Z_{0}^{(4)}(x)-F_{0}(x) \geq 0, \\
& \beta_{0}(x):=V_{0}^{(4)}(x)-F^{0}(x) \leq 0
\end{aligned}
$$

for all $x \in[0, \ell]$.

The iteration process (5.1) can be represented in the form

$$
Z_{p+1}(x)-Z_{p}(x)=\left(\mathcal{T} \alpha_{p}\right)(x), \quad V_{p+1}(x)-V_{p}(x)=\left(\mathcal{T} \beta_{p}\right)(x), \quad x \in[0, \ell],
$$

where

$$
\alpha_{p}(x):=Z_{p}^{(4)}(x)-F_{p}(x), \quad \beta_{p}(x):=V_{p}^{(4)}(x)-F^{p}(x), \quad x \in[0, \ell], p \in \mathbb{N} .
$$

Hence, from (5.3) and (5.4), for any $p \in \mathbb{N} \cup\{0\}$, we obtain

$$
\begin{gathered}
\alpha_{p+1}(x)=F_{p}(x)-F_{p+1}(x), \quad \beta_{p+1}(x)=F^{p}(x)-F^{p+1}(x), \quad x \in[0, \ell], \\
Z_{p}(x)-Z_{p+2}(x)=-\mathcal{T}\left(\alpha_{p}+\alpha_{p+1}\right)(x), \quad x \in[0, \ell], \\
V_{p}(x)-V_{p+2}(x)=-\mathcal{T}\left(\beta_{p}+\beta_{p+1}\right)(x), \quad x \in[0, \ell],
\end{gathered}
$$

and

$$
\begin{aligned}
& \alpha_{p+1}(x)+\alpha_{p+2}(x)=F_{p}(x)-F_{p+2}(x), \quad x \in[0, \ell], \\
& \beta_{p+1}(x)+\beta_{p+2}(x)=F^{p}(x)-F^{p+2}(x), \quad x \in[0, \ell] .
\end{aligned}
$$

Taking into account conditions (4.2), (5.2), and (5.3) with $p=0$, we can see that

$$
\begin{array}{lll}
Z_{1}(x)-Z_{0}(x) \geq 0, & V_{1}(x)-V_{0}(x) \leq 0, & x \in[0, \ell / 3] \cup[2 \ell / 3, \ell], \\
Z_{1}(x)-Z_{0}(x) \leq 0, & V_{1}(x)-V_{0}(x) \geq 0, & x \in[\ell / 3,2 \ell / 3] .
\end{array}
$$


Thus, if $Z_{1}(x), V_{1}(x) \in \mathscr{D}$ for all $x \in\left[\lambda_{0}, \theta_{0}\right]$, then from (5.5) with $p=0$, by virtue of (5.2), (5.8), and (3.1), we obtain $\alpha_{1}(x) \leq 0, \beta_{1}(x) \geq 0$ for all $x \in[0, \ell]$. Therefore, from (4.2) and (5.3) with $p=1$ we get

$$
\begin{aligned}
& Z_{2}(x)-Z_{1}(x) \leq 0, \quad V_{2}(x)-V_{1}(x) \geq 0, \quad x \in[0, \ell / 3] \cup[2 \ell / 3, \ell], \\
& Z_{2}(x)-Z_{1}(x) \geq 0, \quad V_{2}(x)-V_{1}(x) \leq 0, \quad x \in[\ell / 3,2 \ell / 3] .
\end{aligned}
$$

Assume, in addition, that

$$
\alpha_{0}(x)+\alpha_{1}(x) \geq 0, \quad \beta_{0}(x)+\beta_{1}(x) \leq 0, \quad x \in[0, \ell] .
$$

Then from (5.6) with $p=0$ we obtain

$$
\begin{aligned}
& Z_{0}(x)-Z_{2}(x) \leq 0, \quad V_{0}(x)-V_{2}(x) \geq 0, \quad x \in[0, \ell / 3] \cup[2 \ell / 3, \ell], \\
& Z_{0}(x)-Z_{2}(x) \geq 0, \quad V_{0}(x)-V_{2}(x) \leq 0, \quad x \in[\ell / 3,2 \ell / 3],
\end{aligned}
$$

and thus (5.9) and (5.11) result in

$$
\begin{aligned}
Z_{0}(x) \leq Z_{2}(x) \leq Z_{1}(x), \quad V_{1}(x) \leq V_{2}(x) \leq & V_{0}(x), \\
& \text { for } x \in[0, \ell / 3] \cup[2 \ell / 3, \ell],
\end{aligned}
$$

and

$Z_{1}(x) \leq Z_{2}(x) \leq Z_{0}(x), \quad V_{0}(x) \leq V_{2}(x) \leq V_{1}(x) \quad$ for $x \in[\ell / 3,2 \ell / 3]$.

Therefore, we have proved that if $\left\langle Z_{0}, Z_{1}\right\rangle \subseteq \mathscr{D},\left\langle V_{1}, V_{0}\right\rangle \subseteq \mathscr{D}$, and conditions (5.10) hold, then the values $Z_{2}(x)$ and $V_{2}(x)$ of the next approximations which are obtained according to (5.1) also belong to the set $\mathscr{D}$.

From (3.1), (5.10), (5.12), (5.13), and (5.3), (5.5), (5.7) with $p=2,1,0$, we get

$$
\begin{gathered}
\alpha_{2}(x) \geq 0, \quad \beta_{2}(x) \leq 0, \quad x \in[0, \ell], \\
Z_{3}(x)-Z_{2}(x) \geq 0, \quad V_{3}(x)-V_{2}(x) \leq 0, \quad x \in[0, \ell / 3] \cup[2 \ell / 3, \ell], \\
Z_{3}(x)-Z_{2}(x) \leq 0, \quad V_{3}(x)-V_{2}(x) \geq 0, \quad x \in[\ell / 3,2 \ell / 3],
\end{gathered}
$$

and

$$
\alpha_{1}(x)+\alpha_{2}(x) \leq 0, \quad \beta_{1}(x)+\beta_{2}(x) \geq 0, \quad x \in[0, \ell] .
$$

Hence, from (5.6) with $p=1$ we obtain

$$
\begin{aligned}
& Z_{1}(x)-Z_{3}(x) \geq 0, \quad V_{1}(x)-V_{3}(x) \leq 0, \quad x \in[0, \ell / 3] \cup[2 \ell / 3, \ell], \\
& Z_{1}(x)-Z_{3}(x) \leq 0, \quad V_{1}(x)-V_{3}(x) \geq 0, \quad x \in[\ell / 3,2 \ell / 3] .
\end{aligned}
$$

Consequently,

$$
\begin{array}{cc}
Z_{0}(x) \leq Z_{2}(x) \leq Z_{3}(x) \leq Z_{1}(x), & V_{1}(x) \leq V_{3}(x) \leq V_{2}(x) \leq V_{0}(x), \\
& x \in[0, \ell / 3] \cup[2 \ell / 3, \ell], \\
& \\
Z_{1}(x) \leq Z_{3}(x) \leq Z_{2}(x) \leq Z_{0}(x), & V_{0}(x) \leq V_{2}(x) \leq V_{3}(x) \leq V_{1}(x), \\
x & \in[\ell / 3,2 \ell / 3],
\end{array}
$$

and thus $Z_{3}(x), V_{3}(x) \in \mathscr{D}$ for all $x \in\left[\lambda_{0}, \theta_{0}\right]$. 
Using the method of the mathematical induction we can show that if $\left\langle Z_{0}, Z_{1}\right\rangle \subseteq$ $\mathscr{D},\left\langle V_{1}, V_{0}\right\rangle \subseteq \mathscr{D}$, and conditions (5.10) hold, then the sequences $\left\{Z_{p}\right\}_{p=1}^{\infty}$ and $\left\{V_{p}\right\}_{p=1}^{\infty}$, which are constructed according to (5.1), satisfy the inequalities

$$
\begin{aligned}
Z_{2 p}(x) & \leq Z_{2 p+2}(x) \leq Z_{2 p+3}(x) \leq Z_{2 p+1}(x), \\
V_{2 p+1}(x) & \leq V_{2 p+3}(x) \leq V_{2 p+2}(x) \leq V_{2 p}(x)
\end{aligned}
$$

for $x \in[0, \ell / 3] \cup[2 \ell / 3, \ell], p=0,1,2, \ldots$, and

$$
\begin{aligned}
Z_{2 p+1}(x) & \leq Z_{2 p+3}(x) \leq Z_{2 p+2}(x) \leq Z_{2 p}(x), \\
V_{2 p}(x) & \leq V_{2 p+2}(x) \leq V_{2 p+3}(x) \leq V_{2 p+1}(x)
\end{aligned}
$$

for $x \in[\ell / 3,2 \ell / 3], p=0,1,2, \ldots$.

Let us now find a sufficient condition for the uniform, on $\left[\lambda_{0}, \theta_{0}\right]$, convergence of the sequences $\left\{Z_{p}\right\}_{p=1}^{\infty}$ and $\left\{V_{p}\right\}_{p=1}^{\infty}$ to the unique solution of the boundary value problem (2.1)-(2.3).

For any vector $P=\left(p_{i}\right)_{i=1}^{n} \in \mathbb{R}^{n}$, we set

$$
\|P\|:=\max _{i=\overline{1, n}}\left|p_{i}\right| \text {. }
$$

Let us also put

$$
\begin{gathered}
W_{p}(x):=Z_{p}(x)-V_{p}(x), \quad x \in\left[\lambda_{0}, \theta_{0}\right], p=0,1,2 \ldots, \\
\epsilon:=\max _{x \in[0, \ell]}\left\{\left\|Z_{0}(x)-Z_{1}(x)\right\|,\left\|V_{0}(x)-V_{1}(x)\right\|,\left\|W_{0}(x)\right\|\right\},
\end{gathered}
$$

and

$$
d:=\max _{x \in[0, \ell]} \int_{0}^{\ell}|g(x, \xi)| d \xi=\frac{4 \ell^{10}}{3^{7}} .
$$

Then using (5.3), (5.5), we can prove by induction the error estimate

$$
\begin{aligned}
\max _{x \in[0, \ell]}\left\{\left\|Z_{p+1}(x)-Z_{p}(x)\right\|,\left\|V_{p+1}(x)-V_{p}(x)\right\|\right\} & \\
& \leq \epsilon\left(\frac{81}{8 \ell^{6}} d 6\|K\|\right)^{p}=\epsilon\left(\frac{\ell^{4}}{9}\|K\|\right)^{p}
\end{aligned}
$$

valid for all $p \in \mathbb{N}$, where $K$ is the matrix appearing in the Lipschitz condition (3.2) and $\|K\|=\max _{i=\overline{1, n}}\left\{\sum_{j=1}^{n} k_{i j}\right\}$.

If $\|K\|$ satisfies the inequality

$$
\|K\|<\frac{9}{\ell^{4}},
$$

then it follows from estimate (5.14) that the approximations $\left\{Z_{p}\right\}_{p=1}^{\infty}$ and $\left\{V_{p}\right\}_{p=1}^{\infty}$ converge, respectively, to certain limits $Y_{*}$ and $Y^{*}$ uniformly on $\left[\lambda_{0}, \theta_{0}\right]$. 
Let us show that $Y_{*}(x) \equiv Y^{*}(x)$. From (5.1) we have

$$
W_{p+1}(x)= \begin{cases}0 & \text { for } x \in\left[\lambda_{0}, 0\right], \\ \left(\mathcal{T}\left(F^{p}-F_{p}\right)\right)(x) & \text { for } x \in[0, \ell], \\ 0 & \text { for } x \in\left[\ell, \theta_{0}\right] .\end{cases}
$$

It is easy to show that the estimate

$$
\max _{x \in[0, \ell]}\left\|W_{p}(x)\right\| \leq \epsilon\left(\frac{81}{8 \ell^{6}} d 6\|K\|\right)^{p}=\epsilon\left(\frac{\ell^{4}}{9}\|K\|\right)^{p}
$$

is true for $p \in \mathbb{N}$. If condition (5.15) holds, then $\lim _{p \rightarrow \infty} W_{p}(x)=0$ uniformly on $[0, \ell]$, and thus

$$
Y_{*}(x)=Y^{*}(x)=: Y(x), \quad x \in\left[\lambda_{0}, \theta_{0}\right] .
$$

Passing in equalities (5.1) to the limit as $p \rightarrow \infty$, we obtain the equality

$$
Y(x)= \begin{cases}\Phi(x) & \text { for } x \in\left[\lambda_{0}, 0\right], \\ \Omega(x)-(\mathcal{T} \tilde{H})(x) & \text { for } x \in[0, \ell], \\ \Psi(x) & \text { for } x \in\left[\ell, \theta_{0}\right],\end{cases}
$$

where

$$
\begin{aligned}
\tilde{H}(x):=H\left(x, Y(x),\left(\mathcal{g}_{\Lambda} Y\right)\right. & \left.(x),\left(\mathcal{g}_{\Theta} Y\right)(x), Y(x),\left(\mathcal{g}_{\Lambda} Y\right)(x),\left(\mathcal{g}_{\Theta} Y\right)(x)\right) \\
& =F\left(x, Y(x),\left(\mathcal{g}_{\Lambda} Y\right)(x),\left(\mathcal{g}_{\Theta} Y\right)(x)\right), \quad x \in[0, \ell],
\end{aligned}
$$

i. e., $Y$ is a solution of problem (2.1)-(2.3).

The uniqueness of the solution $Y$ under the condition (5.15) can be easily proved by using the Lipschitz condition (3.2).

Consequently, we have proved the following

Theorem. Let $F \in \mathcal{M}_{D}([0, \ell])$ and $Z_{0}, V_{0}$ be comparison functions of problem (2.1)-(2.3) satisfying conditions (5.2). In addition, let the first approximations $Z_{1}$ and $V_{1}$ constructed according to formulae (5.1) be such that $\left\langle Z_{0}, Z_{1}\right\rangle \subseteq \mathscr{D}$, $\left\langle V_{1}, V_{0}\right\rangle \subseteq \mathcal{D}$, and conditions (5.10) hold. Assume also that condition (5.15) is satisfied.

Then the sequences of approximations $\left\{Z_{p}\right\}_{p=1}^{\infty}$ and $\left\{V_{p}\right\}_{p=1}^{\infty}$ constructed according to (5.1) converge uniformly on $\left[\lambda_{0}, \theta_{0}\right]$ to the unique solution $Y$ of problem (2.1)-(2.3) and, moreover,

$$
\begin{aligned}
Z_{2 p}(x) & \leq Z_{2 p+2}(x) \leq Y(x) \leq Z_{2 p+3}(x) \leq Z_{2 p+1}(x), \\
V_{2 p+1}(x) & \leq V_{2 p+3}(x) \leq Y(x) \leq V_{2 p+2}(x) \leq V_{2 p}(x)
\end{aligned}
$$

for $x \in[0, \ell / 3] \cup[2 \ell / 3, \ell], p=0,1,2, \ldots$, and

$$
Z_{2 p+1}(x) \leq Z_{2 p+3}(x) \leq Y(x) \leq Z_{2 p+2}(x) \leq Z_{2 p}(x),
$$




$$
V_{2 p}(x) \leq V_{2 p+2}(x) \leq Y(x) \leq V_{2 p+3}(x) \leq V_{2 p+1}(x)
$$

for $x \in[\ell / 3,2 \ell / 3], p=0,1,2, \ldots$.

Remark. If the domain $D$ is "large" enough, then there exist comparison functions $Z_{0}, V_{0}$ of problem (2.1)-(2.3) satisfying conditions (5.2).

Indeed, let $U:\left[\lambda_{0}, \theta_{0}\right] \rightarrow \mathbb{R}^{n}$ be an arbitrary vector-function which satisfies the boundary conditions (2.2) and the initial condition (2.3) and is such that $\left.U\right|_{[0, \ell]} \in$ $C^{4}\left([0, \ell], \mathbb{R}^{n}\right)$ and $U(x) \in \mathscr{D}$ for all $x \in\left[\lambda_{0}, \theta_{0}\right]$. Then we set

$$
\alpha(x):=U^{(4)}(x)-F\left(x, U(x),\left(\mathscr{g}_{\Lambda} U\right)(x),\left(\mathcal{L}_{\Theta} U\right)(x)\right), \quad x \in[0, \ell] .
$$

It is clear that the problems

$$
\begin{gathered}
\eta^{(4)}=|\alpha(x)|, \\
\eta(0)=0, \quad \eta(\ell / 3)=0, \quad \eta(2 \ell / 3)=0, \quad \eta(\ell)=0
\end{gathered}
$$

and

$$
\begin{gathered}
q^{(4)}=-|\alpha(x)|, \\
q(0)=0, \quad q(\ell / 3)=0, \quad q(2 \ell / 3)=0, \quad q(\ell)=0
\end{gathered}
$$

have unique solutions $\eta$ and $q$, respectively. Relations (4.1) and (4.2) yield

$$
\begin{array}{ll}
\eta(x) \leq 0, & q(x) \geq 0, \quad x \in[0, \ell / 3] \cup[2 \ell / 3, \ell], \\
\eta(x) \geq 0, & q(x) \leq 0, \quad x \in[\ell / 3,2 \ell / 3] .
\end{array}
$$

Now we put

$$
\begin{array}{lll}
Z_{0}(x)=U(x)+\eta(x), & V_{0}(x)=U(x)+q(x), & x \in[0, \ell], \\
Z_{0}(x)=U(x), & V_{0}(x)=U(x), & x \in\left[\lambda_{0}, 0\right] \cup\left[\ell, \theta_{0}\right] .
\end{array}
$$

It is easy to see that $Z_{0}$ and $V_{0}$ satisfy the boundary conditions (2.2), the initial condition (2.3), and inequalities (4.3). If $Z_{0}(x), V_{0}(x) \in \mathscr{D}$ for all $x \in\left[\lambda_{0}, \theta_{0}\right]$, then $Z_{0}, V_{0}$ are comparison functions of problem (2.1)-(2.3) and, using (5.17), (5.18) and assumptions (2a) and (2b) of Section 3, we get

$$
\begin{aligned}
Z_{0}^{(4)}(x) & -F_{0}(x)=U^{(4)}(x)+|\alpha(x)|-F_{0}(x)= \\
& =\alpha(x)+|\alpha(x)|+F\left(x, U(x),\left(\mathcal{g}_{\Lambda} U\right)(x),\left(\mathcal{g}_{\Theta} U\right)(x)\right)-F_{0}(x) \geq 0
\end{aligned}
$$

and

$$
\begin{aligned}
V_{0}^{(4)}(x) & -F^{0}(x)=U^{(4)}(x)-|\alpha(x)|-F^{0}(x)= \\
& =\alpha(x)-|\alpha(x)|+F\left(x, U(x),\left(\mathcal{g}_{\Lambda} U\right)(x),\left(\mathcal{g}_{\Theta} U\right)(x)\right)-F^{0}(x) \leq 0
\end{aligned}
$$

for all $x \in[0, \ell]$. Consequently, $Z_{0}$ and $V_{0}$ also satisfy conditions (5.2). 


\section{REFERENCES}

[1] B. Ahmad, R. Ali Khan, and P. W. Eloe, "Generalized quasilinearization method for a second order three point boundary-value problem with nonlinear boundary conditions," Electron. J. Differential Equations, pp. No. 90, 12 pp. (electronic), 2002.

[2] R. Ali Khan, "The generalized method of quasilinearization and nonlinear boundary value problems with integral boundary conditions," Electron. J. Qual. Theory Differ. Equ., pp. No. 19, 15 pp. (electronic), 2003.

[3] T. Jankowski, "An extension of the method of quasilinearization," Arch. Math. (Brno), vol. 39, no. 3, pp. 201-208, 2003.

[4] A. Luchka, Proekcionno-iterativnye metody [Projection-iteration methods]. Kiev: "Naukova Dumka", 1993, in Russian.

[5] V. V. Marinets, "On an approach to construction of iteration methods for approximate integration of boundary value problems arising in the theory of plates and hulls," in Proceedings of the VIII All-Union Conference "Numerical Methods for Solution of Problems of Elasticity and Plasticity Theory", Novosibirsk, 1984, pp. 194-198, in Russian.

[6] V. V. Marinets and O. O. Shomodi, "Two-sided methods of integrations of boundary-value problems," Nauk. Visnyk Uzhgorod Nat. University, Ser. Mat. Inform., vol. 4, pp. 63-74, 2000.

[7] M. A. Naimark, Linear differential operators. Part I: Elementary theory of linear differential operators. New York: Frederick Ungar Publishing Co., 1967.

[8] M. A. Naı̆mark, Linear differential operators. Part II: Linear differential operators in Hilbert space, ser. With additional material by the author, and a supplement by V. È. Ljance. Translated from the Russian by E. R. Dawson. English translation edited by W. N. Everitt. New York: Frederick Ungar Publishing Co., 1968.

[9] A. Qi and Y. Liu, "Monotone iterative techniques and a periodic boundary value problem for first order differential equations with a functional argument," Georgian Math. J., vol. 7, no. 2, pp. 373-378, 2000.

[10] M. Ronto and A. M. Samoilenko, Numerical-analytic methods in the theory of boundary-value problems. River Edge, NJ: World Scientific Publishing Co. Inc., 2000, with a preface by Yu. A. Mitropolsky.

[11] A. M. Samoĭlenko and N. I. Ronto, Chislenno-analiticheskie metody issledovaniya reshenii kraevykh zadach. Kiev: "Naukova Dumka", 1986, with an English summary, Edited and with a preface by Yu. A. Mitropol'skiı̌.

\section{Author's address}

\section{O. Pytovka}

State University of Mukachevo, 26 Uzhgorodska St., Mukachevo, Ukraine

E-mail address: oxana_pityovka@bigmir.net 